\title{
Modeling and prediction of cell population dynamics
}

\author{
Youngil Lim \\ ${ }^{a}$ Department of chemical engineering, Hankyong National University \\ Kyonggi-do Ansung-si Sukjung-dong 67, 456-749 Korea
}

\begin{abstract}
Oscillatory yeast cell dynamics are observed in glucose-limited growth environments. Under such conditions, both glucose and the excreted product ethanol can serve as substrates for cell growth. The cell dynamics is described by a PDE (partial differential equation) system containing one PDE for the cell population and 8 ODEs for 8 substrates variations (extracellular glucose, extracellular ethanol, intracellular glucose, intracellular ethanol, exhausted oxygen, exhausted carbon dioxide, dissolved oxygen and dissolved carbon dioxide).

The system is solved by the numerical method of characteristics (MOC) modified (Lim et al., 2002). Here, mesh points are added at the smallest cell size and also deleted at the largest cell size at the same time level. The modified MOC provides a solution free of numerical dissipation error caused by the cell growth term (i.e., convection term), owing to the mass axis moving along a cell growth pathline.

The oscillatory behavior of the cell number and the cell mass is predicted from the simulation. The cell number variation affects the extracellular glucose/ethanol and oxygen/carbon-dioxide concentrations in the gas exhaust stream. As ethanol is excreted primarily by budded cells, it is shown for the extracellular ethanol concentration to slowly reach a regular oscillatory state. Dynamics of the evolved oxygen concentration ratio and carbon-dioxide concentration ratio are also shown.
\end{abstract}

Keywords: Yeast cell culture, Cell population dynamics, Population balance equation, Cell oscillatory behavior, Modified method of characteristics

\section{Introduction}

Cell cultures are composed of discrete microorganisms whose population dynamics play an important role in bioreactor design and control. The cell cultures are known to exhibit autonomous oscillations that affect bioreactor stability and productivity. To increase the productivity and stability, it is therefore desirable to derive a dynamic model that describes the oscillatory behavior and to develop a control strategy that allows modification of such intrinsic reactor dynamics (Henson, 2003).

\footnotetext{
${ }^{a}$ Author/s to whom correspondence should be addressed: Tel: +82 31670 5207, Fax: +82 31670 5015, Email: limyi@hknu.ac.kr.
} 
As an exa mple of the dynamic model of cell cultures, take a segregated/unstructured model based on the cell population balance equation (PBE) coupled to metabolic reactions which are relevant for the extracellular environment (Zhu et al., 2000; Mhaskar et al., 2002). The segregated/unstructured model provides a realistic description of the cell cycle events that lead to sustained oscillation in cell cultures under the assumption that oscillations arise as a result of interactions between the cell population and the extracellular environment. The cell population dynamics including cell growth and cell division is described by a partial differential equation (PDE) including a growth term, newborn-cell birth term, mother-cell division death term and dilution loss.

Oscillatory yeast dynamics are observed in glucose-limited growth environments. Under such conditions, both glucose and the excreted product ethanol can serve as substrates for cell growth. The substrate dynamics is coupled to the cell population balance equation.

A number of numerical methods to solve the cell population balance equation are presented recently; finite difference methods (Mantzaris et al., 2001a), finite element methods (Mantzaris et al., 2001b; Mhaskar et al., 2002). In this study, the system i solved by the numerical method of characteristics (MOC) modified (Lim et al., 2002). Here, mesh points are added at the smallest cell size and also deleted at the largest cell size at the same time level. The modified MOC provides a solution free of numerical dissipation error caused by the cell growth term (i.e., convection term), using the mass axis moving along a cell growth pathline.

In this study, the oscillatory behavior of the cell number and the cell mass are predicted from the simulation. The cell number variation affects the extracellular glucose/ethanol and oxygen/carbon-dioxide concentrations in the gas exhaust stream.

\section{Cell population dynamics}

The cell population dynamics including cell growth and cell division is described by a partial differential equation including a convection term $\left(\frac{\partial v_{g} W}{\partial m}\right)$, newborn-cell birth term $\left(\int 2 p \Gamma W d m\right)$, mother-cell division death term $(-\Gamma W)$ and dilution loss $(-D W)$ : $\frac{\partial W}{\partial t}=-\frac{\partial\left(v_{g}\left(S^{\prime}\right) \cdot W\right)}{\partial m}+\int_{0}^{m^{\prime}} 2 p\left(m, m^{\prime}\right) \Gamma\left(m^{\prime}, S^{\prime}\right) W\left(m^{\prime}, t\right) d m^{\prime}-[D+\Gamma(m)] W$

where $W(m, t)$ is the cell number concentration (no./g) as a function of mass (m) and time $(\mathrm{t}), v_{g}\left(S^{\prime}\right)$ is the overall single cell growth rate at the substrate concentration $\left(S^{\prime}=G^{\prime}+E^{\prime}\right), p\left(m, m^{\prime}\right)$ is the newborn-cell mass distribution function with newborncell mass $(m)$ and mother-cell mass $m^{\prime}, \Gamma\left(m^{\prime}, S^{\prime}\right)$ is the division intensity function, and $D$ is the dilution rate. Note that the PBE based on the number $(N(m, t))$ is often preferred, for conservation of the mass and the number of crystals, in the cases involving agglomeration and breakage kinetics. Thus, Eq. (1) can be modified by (Lim et al., 2002; Mantzaris et al., 1999): 
$\frac{\partial N}{\partial t}=-\frac{\partial\left(v_{g}\left(S^{\prime}\right) \cdot N\right)}{\partial m}+\int_{0}^{m^{\prime}} 2 p\left(m, m^{\prime}\right) \Gamma\left(m^{\prime}, S^{\prime}\right) N\left(m^{\prime}, t\right) d m^{\prime}-[D+\Gamma(m)] N$

On the basis of the number PBE Eq. (2), the eight substrates variations (intracellular glucose, intracellular ethanol, extracellular glucose, extracellular ethanol, exhausted oxygen, exhausted carbon dioxide, dissolved oxygen, dissolved carbon dioxide) are modeled as:

$\frac{d G^{\prime}}{d t}=k_{g}\left(G-G^{\prime}\right)$

$\frac{d E^{\prime}}{d t}=k_{e}\left(E-E^{\prime}\right)$

$\frac{d O}{d t}=k_{o} a\left(O^{*}-O\right)-\left(\frac{192}{180} \frac{K_{g o}\left(G^{\prime}\right)}{Y_{g o}}+\frac{96}{46} \frac{K_{e o}\left(E^{\prime}\right)}{Y_{e o}}\right) N_{\text {total }}$

$V_{g} \frac{d O_{\text {out }}}{d t}=F\left(O_{\text {in }}-O_{\text {out }}\right)-k_{o} a\left(O^{*}-O\right) \cdot V_{l}$

$\frac{d G}{d t}=D\left(G_{f}-G\right)-\left(\frac{K_{g f}\left(G^{\prime}\right)}{Y_{g f}}+\frac{K_{g o}\left(G^{\prime}\right)}{Y_{g o}}\right) N_{t o t a l}$

$\frac{d E}{d t}=D\left(E_{f}-E\right)+\frac{92}{180} \frac{K_{g f}\left(G^{\prime}\right)}{Y_{g f}} N_{\text {total }}^{\text {ethanol }}-\frac{K_{e o}\left(E^{\prime}\right)}{Y_{\text {eo }}} N_{\text {total }}$

$\frac{d C}{d t}=k_{c} a\left(C^{*}-C\right)+\left(\frac{264}{180} \frac{K_{g o}\left(G^{\prime}\right)}{Y_{g o}}+\frac{88}{46} \frac{K_{e o}\left(E^{\prime}\right)}{Y_{e o}}\right) N_{\text {total }}+\frac{88}{180} \frac{K_{g f}\left(G^{\prime}\right)}{Y_{g f}} N_{\text {total }}^{\text {ethanol }}$ (9)

$V_{g} \frac{d C_{\text {out }}}{d t}=F\left(C_{\text {in }}-C_{\text {out }}\right)-k_{c} a\left(C^{*}-C\right) \cdot V_{l}$

where $G^{\prime}, E^{\prime}$ are intracellular glucose and ethanol concentrations; $G, E$ are extracellular concentrations of glucose and ethanol; liquid oxygen concentrations $(O)$; oxygen concentration in the gas exhaust stream $\left(O_{\text {out }}\right)$; liquid phase carbon dioxide concentration $(C)$; gas phase $\mathrm{CO}_{2}$ concentration $\left(\mathrm{C}_{\text {out }}\right)$. Detail models and their parameters are given in Mhaskar et al. (2002) for a S. cerevisiae (or yeast) culture. In summary, this cell PBE model is expressed as a PDE system containing one PDE for the cell number population $(N(m, t))$ and 8 ODEs for 8 substrates variations $\left(G, E, G^{\prime}, E^{\prime}, O, C, O_{\text {out }}\right.$, and $C_{\text {out }}$ ). For convenience, all of the masses have the units $\left[\times 10^{-13} \mathrm{~g}\right]$ and the cell number concentration $(N(m, t))$ has the units $\left[\times 10^{13} n o.\right]$. Fortunately, this scalar PBE model can be solved by the method of characteristics (MOC), because there is a unique growth rate $\left(v_{g}\left(S^{\prime}\right)\right)$ in Eq. (2). If due to several PBEs there are different growth rates in a system, the MOC is not applicable to this kind of the problems .

\section{Modified method of characteristics}

It is well known that for the scalar linear conservation law (e.g., population balance equation mentioned here) there usually exists a unique characteristic curve along which 
information propagates. If the solution moves along the pathline of propagation, the convection term $\frac{\partial\left(v_{g} N\right)}{\partial m}$ in the PBE disappears. Hence, numerical error and instability caused by approximation of the convection term is removed. The MOC has often been used to obtain analytic solution of hyperbolic integro-PDEs (Kumar \& Ramkrishna, 1997).

The MOC formulation for the PBE is expressed as:

$\frac{\partial N(m, t)}{\partial t}=\int_{0}^{m^{\prime}} 2 p\left(m, m^{\prime}\right) \Gamma\left(m^{\prime}, S^{\prime}\right) N\left(m^{\prime}, t\right) d m^{\prime}-[D+\Gamma(m)] N(m, t)$

$\frac{d m}{d t}=v_{g}\left(S^{\prime}\right)$

where $\mathrm{N}(\mathrm{m}, \mathrm{t})$ is the cell number with the units [no.]. The second equation of Eq. (11) is the mesh movement equation. The eight ODEs remain same. The MOC formulation is numerically solved by using a standard method of lines after spatial discretization. Since the mass axis moves continuously as cell growth rate, the mass corresponding to newborn cells can be excluded. To overcome the mass exclusion of new-born cells in the MOC, a new mesh of the smallest mass size $\left(m_{1}\right)$ is added at given time levels. The system size can keep constant by deleting the last mesh at the same time levels (Lim et al., 2002). Since the number of new-born cells can be affected by the number of mesh points added, a proper number of added mesh points should be selected according to stiffness of the system. A simple method for the adaptive time step is proposed as:

$(\Delta t)_{i+1}=\frac{\Delta \bar{m}}{\left(v_{g}\right)_{i}}$

where the next time stepsize $(\Delta t)_{i+1}$ when a smallest mesh point will be inserted is determined by the average mesh stepsize $\Delta \bar{m}$ divided by the cell growth rate $\left(v_{g}\right)_{i}$ at the present time level.

\section{Numerical results}

Fig. 1 shows dynamics of the cell number $(N(m, t))$ solved by the numerical MOC on 102 mesh points ( $N_{\text {mesh }}=102$ ) where the first and last mesh points is added and deleted respectively 101 times. Due to cell division, the cell number density of small sizes tends to increase with time and the oscillatory behavior of the cell number density are regularized after about $t=8 h r$.

The oscillatory behavior of the cell number $\left(N_{\text {total }}=\int W(m, t) d m=\sum N_{i}\right)$ and the cell mass are shown in Fig. 2 (a)-(b). The cell number variation affects the extracellular glucose/ethanol ( $G$ and $E$ ) and oxygen/carbondioxide $\left(O_{\text {out }}\right.$ and $C_{\text {out }}$ ) concentrations in the gas exhaust stream (see Fig. 2 (c)-(f), respectively). It is shown in Fig. 2 (d) that the extracellular ethanol concentration $(E)$ reaches an oscillatory state. Fig. $2(\mathrm{~g})-(\mathrm{h})$ depict dynamics of the evolved oxygen concentration ratio $100 \times\left(\frac{O_{\text {in }}-O_{\text {out }}}{O}\right)$ and carbondioxide concentration ratio $100 \times\left(\frac{C_{\text {in }}-C_{\text {out }}}{C}\right)$, respectively. 


\section{Conclusion}

Oscillatory dynamics of the cell population are modeled by the population balance equation (PBE) including substrate concentration kinetics. For the cell number conservation, the cell population is described by the number of cells instead of the number density. The model is solved by the numerical method of characteristics (MOC) modified in a sense that the first mesh point is updated adaptively.

The oscillatory behaviors of the cell number and mass are observed. Due to these oscillatory behaviors, the intracellular and extracellular substrates show cyclic profiles at the same cycle time as that of the cell number and mass.

\section{References}

Henson, M.A., 2003, Dynamic modeling of microbial cell populations, Current Opinion in Biotechnology, 14, 460.

Kumar, S. and D. Ramkrishna, 1997, On the solution by discretization balance equations by discretization-III. Nucleation, growth and aggregation of particles, Chem. Eng. Sci., 52(24), 4659.

Lim, Y.I., J.M. Le Lann, X.M. Meyer, X. Joulia, G.B. Lee and E.S. Yoon, 2002, On the solution of Population Balance Equations (PBE) with accurate front tracking methods in practical crystallization processes, Chem. Eng. Sci., 57, 177.

Mantzaris, N.V., J.L. Liou, P. Daoutidis and F. Srienc, 1999, Numerical solution of a mass structured cell population balance model in an environment of changing substrate concentration, J. Biotech., 71, 157.

Mantzaris, N.V., P. Daoutidis and F. Srienc, 2001a, Numerical solution of multivariable cell population balance models: I. Finite difference methods, Computers and Chemical Engineering, 25, 1411.

Mantzaris, N.V., P. Daoutidis and F. Srienc, 2001b, Numerical solution of multivariable cell population balance models: III. Finite element methods, Computers and Chemical Engineering, 25, 1463.

Mhaskar, P., M.A. Hjortsø and M.A. Henson, 2002, Cell population modeling and parameter estimation for continuous cultures of S. cerevisiae, Biotechnol. Prog., 18, 1010.

Zhu, G.-Y., A. Zamamiri, M.A. Henson and M.A. Hjortsø, 2000, Model predictive control of continuous yeast bioreactors using cell population balance models, Chem. Eng. Sci., 55, 6155

\section{Acknowledgements}

This work is financially supported by GRRC (Gyeong-gi Regional Research Center) at Hankyong National University, Korea. 


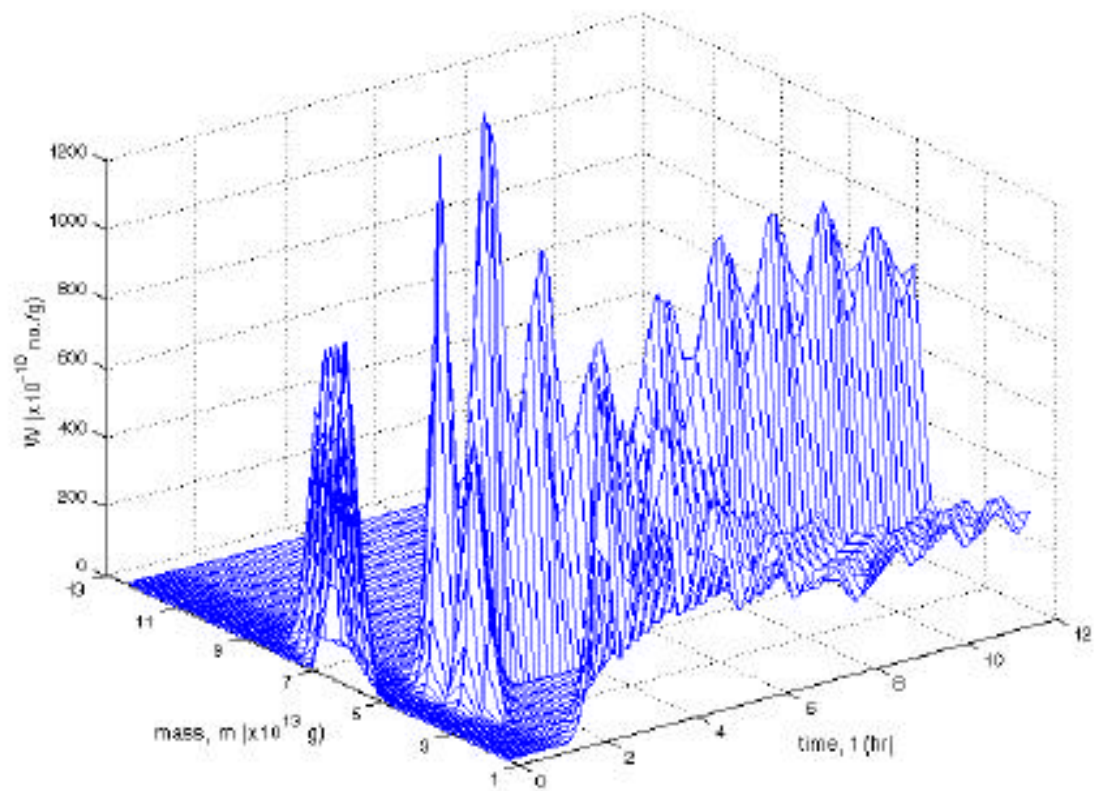

Fig. 1 Distribution of cell population concentration $(W(m, t)$ over mass and time.
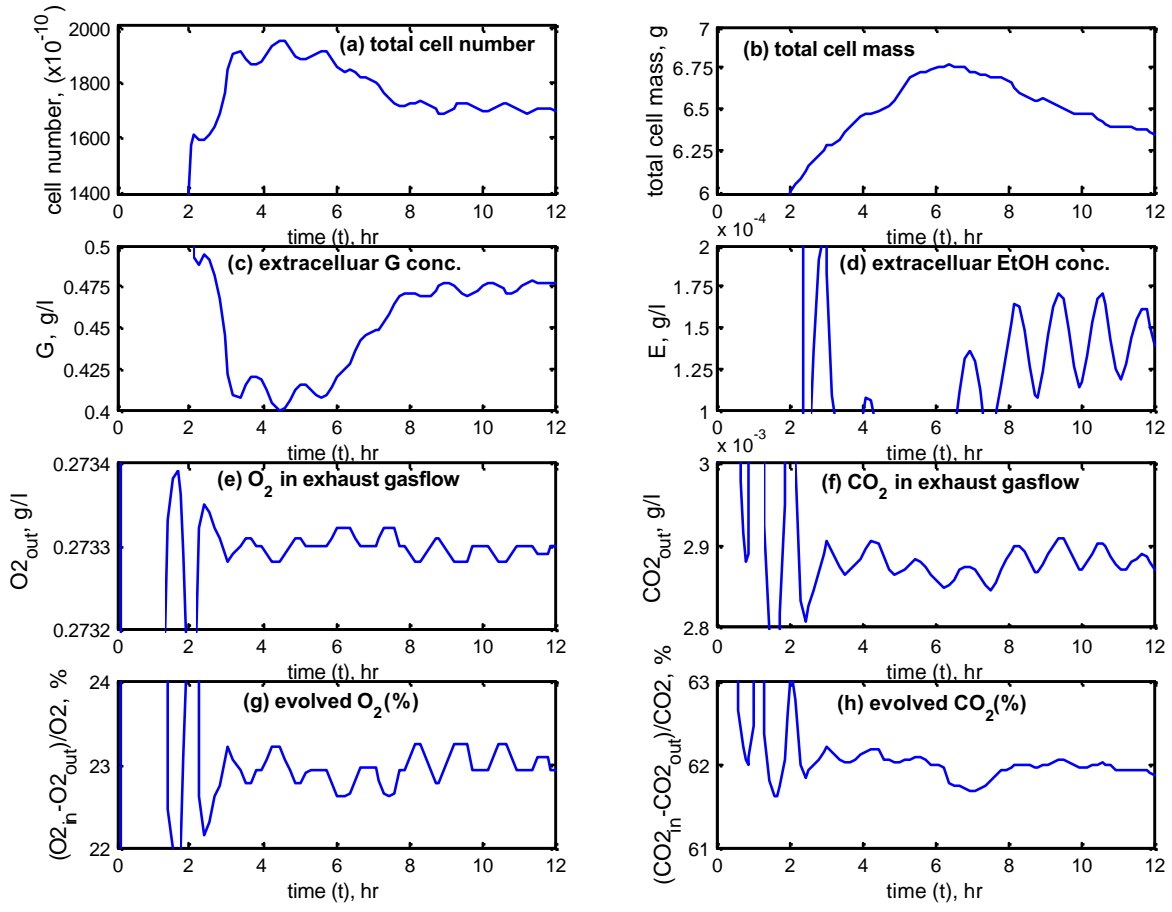

Fig. 2 Oscillatory behavior in time of (a) cell number, (b) cell mass, (c) extracellular glucose $(\mathrm{G}),(\mathrm{d})$ extracellular ethanol $(\mathrm{E}),(\mathrm{e})$ oxygen in exhaust gas stream $\left(O_{\text {out }}\right)$, (f) carbondioxide in exhaust gas stream $\left(C_{\text {out }}\right),(\mathrm{g})$ evolved oxygen ratio and $(\mathrm{h})$ evolved carbondioxide ratio. 